\title{
SMASIS2018-8165
}

\section{DRAFT: EXPERIMENTAL VALIDATION OF ADAPTIVE CONTROL FOR A SHAPE MEMORY ALLOY ACTUATED LIGHTWEIGHT ROBOTIC ARM}

\author{
Serket Quintanar-Guzmán* \\ SnT \\ University of Luxembourg \\ UIT Longwy \\ Université de Lorraine \\ Email: serket.quintanar@uni.lu
}

\author{
Somasundar Kannan, \\ Holger Voos \\ SnT \\ University of Luxembourg \\ Luxembourg, 1855 \\ Luxembourg
}

\author{
Mohamed Darouach, \\ Marouane Alma \\ IUT Longwy \\ Université de Lorraine \\ Cosnes-et-Romai, 90041 \\ France
}

\begin{abstract}
This article presents the experimental validation of a Direct Adaptive Control for angular position regulation of a lightweight robotic arm. The robotic arm is single degree-of-freedom (DOF) system, actuated by two Shape Memory Alloy (SMA) wires. The proposed adaptive control is capable of adapting itself to the hysteretic behavior of SMA wires and update its behavior to deal with the changing parameters of the material over time. The closed-loop approach is tested experimentally showing its effectiveness to deal with the highly nonlinear dynamics of the SMA wires. These results are discussed and compared with a classical control approach. The updated design and hardware development and modeling of the robotic arm are shown.
\end{abstract}

\section{INTRODUCTION}

In recent years, Shape Memory Technology (SMT) has become a trend in the research for alternative actuation systems. The term SMT is used to describe the implementation of materials with Shape Memory Effect (SME). This effect is the property of materials to recover their original shape, after being deformed, upon external stimuli. These stimuli can be thermal, chemical, mechanical, among others. A common type of materials with SMA is the Shape Memory Alloys (SMA). These materials include a group of alloys (most commonly Nickel-

\footnotetext{
*Address all correspondence to this author.
}

Titanium [1]) which can be easily deformed at lower temperatures and then recover their original shape when subject to proper mechanical or thermal stimuli. This transformation occurs due to an inner shifting in the material's crystalline structure. At lower temperatures, the material transforms into martensite, a highly malleable phase. When the SMA is subjected to the proper external stimuli, it transforms into austenite phase, a rigid cubic structure, allowing the material to recover its original shape [2].

The increased interest in the implementation and research of these materials is due to their multiple advantages over conventional actuators, advantages such as high force to mass ratio, corrosion resistance, biocompatibility, noiseless operation, among others. These advantages make SMA suitable for a wide range of applications, ranging from biomedical and dental implants to aerospace engineering. Some SMA applications that we can find in the literature are for example, in medical areas as intra-arterial supports [3], adaptive anklefoot orthoses [4], skeletal fixation devices [5] or orthodontic applications [6]. We can also find implementations in aerodynamics as an actuator for morphing segments on UAV [7] or wing shape control [8].

Many other specific purpose applications have been reported in the literature such as camera lens focus actuators [9], car mirror actuators [10] or SMA based motors [11]. Other examples of this are the multiple robotics implementations. For instance, the robotic arm developed by the author in [12] or the anthropomorphic robotic fingers proposed by [13] and [14]. Along with
Copyright (C) 2018 by ASME 
these specific purpose applications some more general, advanced control oriented application have been developed as the ones presented in [15-18].

In spite of their multiple advantages, SMAs also entail different kind of challenges for mechanism design and the control of them. Most of the aforementioned applications, imply a complicated mechanical design or are oriented to micro-scale actuation. To solve this problem, a lightweight design for an SMA actuated robotic arm was proposed in [19]. This proposal, using 3D printing technology and lightweight materials, together with a relatively simple mechanical design, achieves a lightweight robotic arm suitable for implementation in mobile environments where the weight and size is a primary concern, as flying manipulation. An improved mechanical design of this proposal is introduced in the current work.

In addition to the mechanical challenges, the nonlinear dynamics of the SMAs make them difficult to control and model. Among these nonlinear dynamics, we found principally a highly hysteretic behavior, phenomena as dead zone or superelasticity. For this reason, multiple control approaches have been developed an reported in the literature. From simple classical control techniques as PID or Variable Structure Control (VSC) [20], to intelligent control as fuzzy control approach [13] or neural networks [12]. A set of techniques proven to be suitable to face the highly nonlinear behavior of these materials are those of the adaptive control. Different adaptive techniques have been implemented for SMA control. These techniques can be broadly classify in Direct and Indirect adaptive control [15-17, 21, 22] and intelligent adaptive control. This latter class includes techniques combining adaptive and intelligent control, like the ones reported in [23-25]. These intelligent adaptive controls depend on the identification of multiple parameters, and their quality relies on the number of neurons and persistent excitation conditions. Contrary to these methods, we propose a Direct adaptive control method where only one parameter needs to be tuned in real-time for each SMA wire.

\section{SMA WIRE ACTUATED ROBOT ARM DESIGN}

This section presents the mechanical design of the proposed SMA wire actuated robotic arm. Figure 1 shows a Computer Aided Design (CAD) model of the lightweight robotic arm. The mechanism presented here is based on a previous work published in [26,27]. The proposed design is a lightweight robotic arm with one Degree of Freedom (DOF), actuated by 3 SMA wires. The custom design of the pieces, together with 3D printing technology and light fabrication materials, permit the construction of a mechanism with an approximated weight of $50 \mathrm{~g}$ and a range of angular displacement up to 90 degrees.

The robotic arm presented in $[26,27]$ is originally based on the joint proposed by the authors in [20]. This design proposes two couplers (coupler-1 and coupler-2), each one actuated by an individual SMA wire (SMA-1 and SMA-2 respectively), and joined with a torsion spring in between the couplers. This configuration allows for faster response of the overall system by controlling the total stiffness of the joint, thus increasing or decreasing the transformation temperatures, which leads to a faster forward or backward transformation of the SMA-1. The stiffness adjustment is carried out by the SMA-2 while the angular position of the joint, and the end-effector's position, depends directly on the SMA-1. The current work proposes a new mechanical design for the robotic arm's prototype. A couple of groove bearings are implemented, substituting the previously proposed winding wheels (see [27]). This change allows lower overall friction, thus higher angular displacements while handling the SMA wires relative high temperatures adequately. Furthermore, an entirely new mechanical design for the gripper and its actuation system is presented. This end-effector is actuated by a biased SMA wire. The bias force is provided by a 3D-printed custommade spring. The SMA wire is wound over the spring itself. The later one converts the transversal movement of the wire into longitudinal movement along the shaft of the end-effector, which induces the open-close motion of the gripper.

The low weight of the overall actuator, along with its wide range rotational displacement capability, makes this actuator a suitable alternative for applications in aerial manipulation with small unmanned aerial vehicles (UAVs). Although all the experimental test presented in this work were performed with a wired communication, the proposed system is equipped for wireless communication using Robot Operating System (ROS). ROS implementation allows for future mobile environment tests, as a flying manipulator on UAVs for example.

\section{SYSTEM MODEL}

In this section, the mathematical model of the SMA wire actuated robotic arm is briefly described. Figure 2 shows a block diagram of the full robotic arm model. The model consists of three elementary submodels: 1) SMA wire model, 2) Kinematic model and 3) Dynamic model. These are briefly described in the next subsections.

\section{SMA wire model}

The SMA wire model is subdivided into three submodels as shown in Figure 3. The first submodel is the heat transfer model which describes the thermal dynamics of the SMA wire when being heated by Joule effect and cooled down by natural convection. The next submodel, the phase transformation model, computes the SMA wires' martensite fraction $(\xi)$. To accurately represent the characteristic hysteretic behavior of the SMA wire, this model consists of two different equations for cooling and heating respectively. Finally, the constitutive model describes the dynamic relation among stress $(\sigma)$, strain $(\varepsilon)$, temperature 


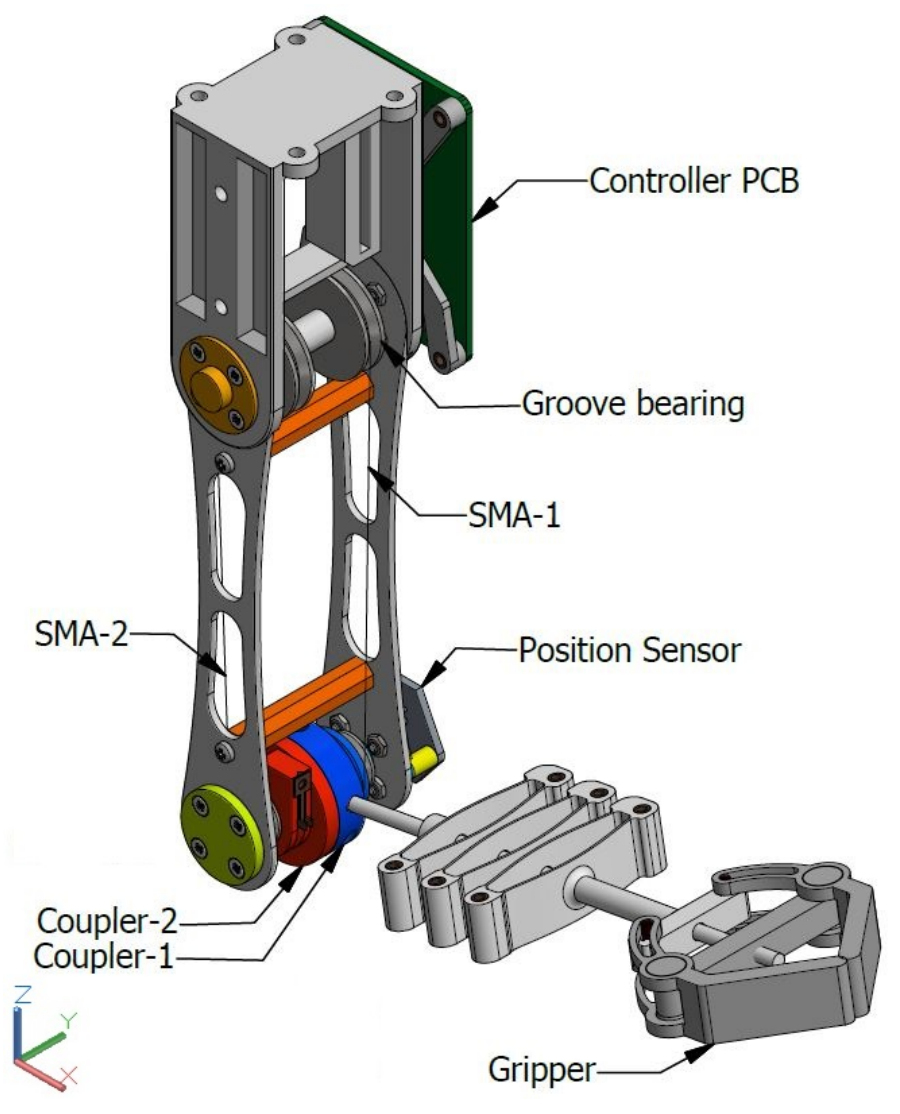

FIGURE 1: PROPOSED ROBOT ARM CAD MODEL

$(T)$ and martensite fraction $(\xi)$. This model allows describing the relationship between the SMA wire's dynamic and the dynamics of the robotic arm. This constitutive model was developed in [28] and then improved in [29].

\section{Kinematic model}

The kinematic model is the bridge between the SMA model and the dynamic model. This model describes the relation between the strain of the SMA wire and the angular position of the coupler with respect to $Z$-axis. The kinematic model depends directly on the geometric and mechanical design of the robotic arm mechanism. This relation is expressed as:

$$
\dot{\varepsilon}_{i}=-\frac{r_{i} \dot{\theta}_{i}}{l_{0 i}}
$$

where $r$ is the coupler radius, $l_{0}$ the initial length of the SMA wire and $\dot{\theta}$ the angular velocity of the coupler to which the SMA wire is attached. Finally the sub-index $i=1,2$ indicates the corresponding wire SMA- $i$.

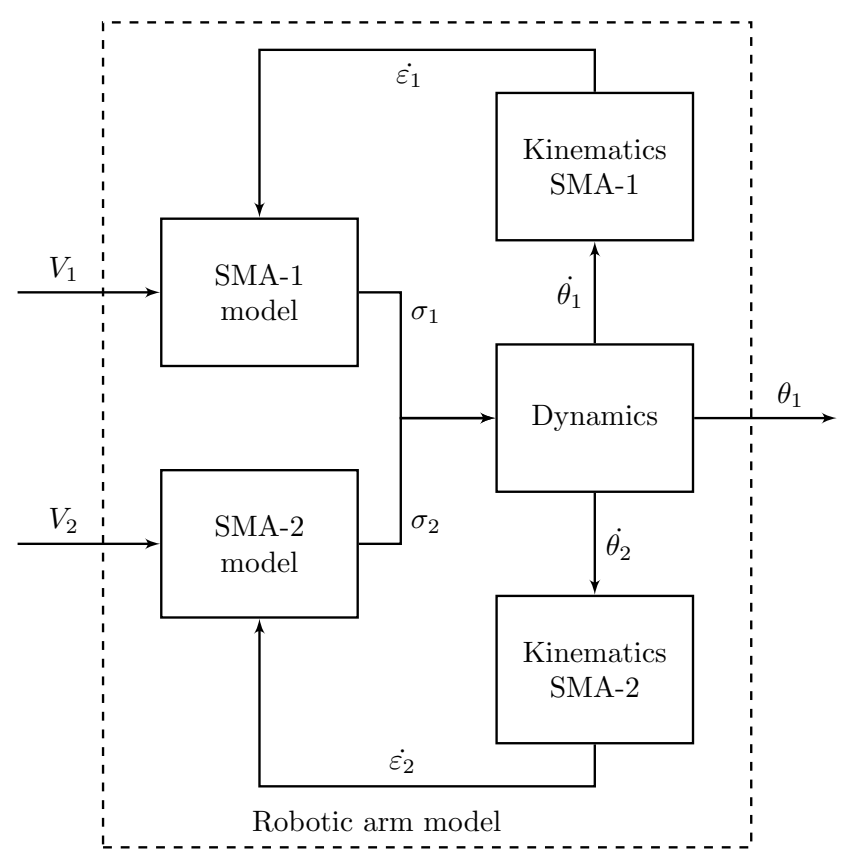

FIGURE 2: ROBOTIC ARM BLOCK DIAGRAM

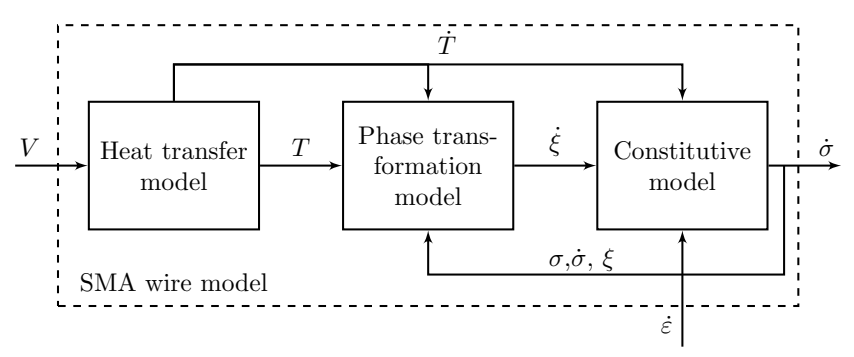

FIGURE 3: SMA WIRE BLOCK DIAGRAM

\section{Dynamic model}

The dynamic model describes the interaction among the couplers mechanism, torsion spring, SMA wires and other phenomena like gravity and friction. In general terms, the dynamic model for a robotic arm is written as follows:

$$
I(\theta) \ddot{\theta}+C_{m}(\theta, \dot{\theta}) \dot{\theta}+g(\theta)+V_{d} \dot{\theta}+\Phi\left(\theta, \theta_{r}\right)=\tau_{\omega}
$$

where $\theta, \dot{\theta}, \ddot{\theta}$ are the angular position, velocity and acceleration of coupler-1 respectively, $I(\theta)$ represents the inertia matrix, $C_{m}(\theta, \dot{\theta})$ is the centripetal-coriolis matrix, $g(\theta)$ is the gravity effect over the system, $V_{d}$ is the dynamic friction coefficient matrix, $\Phi\left(\theta, \theta_{r}\right)$ describes the hysteresis present in the system, $\tau_{\omega}$ is the torque generated by the stress applied by the SMA wires 
over each coupler. This torque can be expressed as:

$$
\tau_{w i}=F_{w i} r_{i}=A_{i} \sigma_{i} r_{i}
$$

where $F_{w i}$ is the SMA-i wire mechanical force.

For further detail on the mathematical model of the full SMA wire actuated robotic arm please refer to [26].

\section{ADAPTIVE CONTROL FOR POSITION REGULATION}

In this section, the analytical development of a Direct Adaptive Control for the two SMA wires actuated robotic arm is presented. This proposal is based on the adaptive control presented by the authors in $[21,22]$. The experimental validation of this controller is presented in the following section. This adaptive approach considers the general dynamic model for a robotic arm shown in Eqn. (2).

Defining the angular position error $(e)$ as follows:

$$
e=\theta_{r}-\theta
$$

where $\theta_{r}$ is the desired angular position of the end-effector. And the first time derivative of the error is given by:

$$
\dot{e}=\frac{d}{d t} e
$$

Subsequently we denote the filtered error $r(t)$ as:

$$
r(t)=\dot{e}(t)+\alpha e(t)
$$

where $\alpha$ is a known positive gain, and each SMA wire has independent filtered errors. The robotic arm's open-loop, after mathematical manipulation, can be written as [30]:

$$
I(\theta) \dot{r}=-C_{m}(\theta, \dot{\theta}) r+\varsigma-\tau
$$

and

$$
\begin{aligned}
& \varsigma=I(\theta)\left(\ddot{\theta}_{r}+\alpha \dot{e}\right)+C_{m}(\theta, \dot{\theta})\left(\dot{\theta}_{r}+\alpha e\right) \\
&+g(\theta)+V_{d} \dot{\theta}+\Phi\left(\theta, \theta_{r}\right)
\end{aligned}
$$

Based on Eqn. (7), the control input is chosen as:

$$
\tau=\hat{\varsigma}+K r
$$

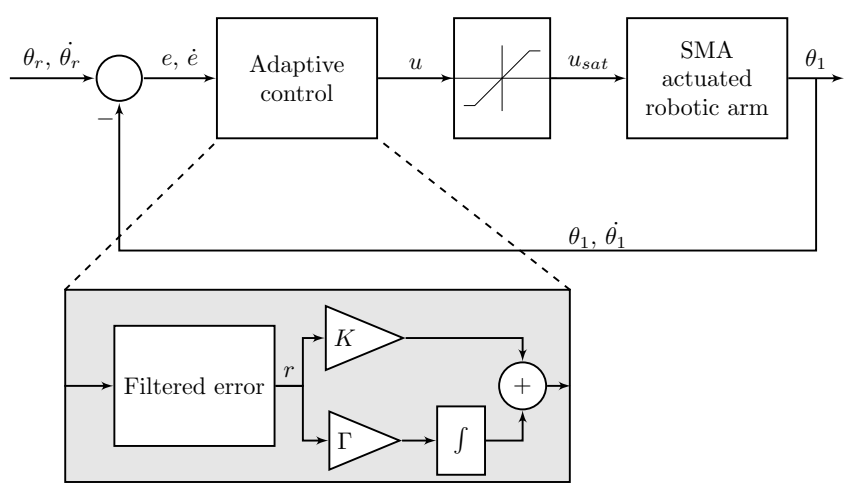

FIGURE 4: ADAPTIVE CONTROL BLOCK DIAGRAM.

where $\tau$ is the control input vector, $K$ is a positive gain matrix and $\hat{\zeta}$ is the estimated value of $\zeta$. $\hat{\zeta}$ is estimated as:

$$
\hat{\zeta}=\Gamma^{-1} r
$$

where $\varphi$ is a positive adaptive gain. The closed-loop dynamic is written then as follows:

$$
I(\theta) \dot{r}=-C_{m}(\theta, \dot{\theta}) r+K r+\tilde{\zeta}
$$

and $\tilde{\boldsymbol{\zeta}}=\boldsymbol{\varsigma}-\hat{\boldsymbol{\zeta}}$. For further details on the development of this control approach and stability analysis please refer to [21,22].

A saturation step is applied to the control input obtained from the proposed adaptive control (AC). This saturation avoids overheating of the SMA wire as well as negative voltages. The full block diagram of this control scheme is shown in Fig. 4.

For comparative analysis, a PID control is also designed and experimentally tested. Defining the regulation error as shown in Eqn. (4), the PID control law is computed as:

$$
u_{i}(t)=K_{p i} e(t)+K_{i i} \int e(t) d t+K_{d i} \dot{e}(t)
$$

Similar to the AC control the PID control signal is also limited to avoid overheating of the SMA wire or negative voltages.

\section{EXPERIMENTAL RESULTS}

This section presents the comparison and discussion of the experimental results of both control approaches namely AC and PID. Figure 5 shows a block diagram of the experimental setup designed for the results presented in this section. An image of the actual experimental setup is displayed in Fig. 6.

The control input for both control approaches (AC and PID) is computed in Matlab/Simulink. This voltage value is sent via 


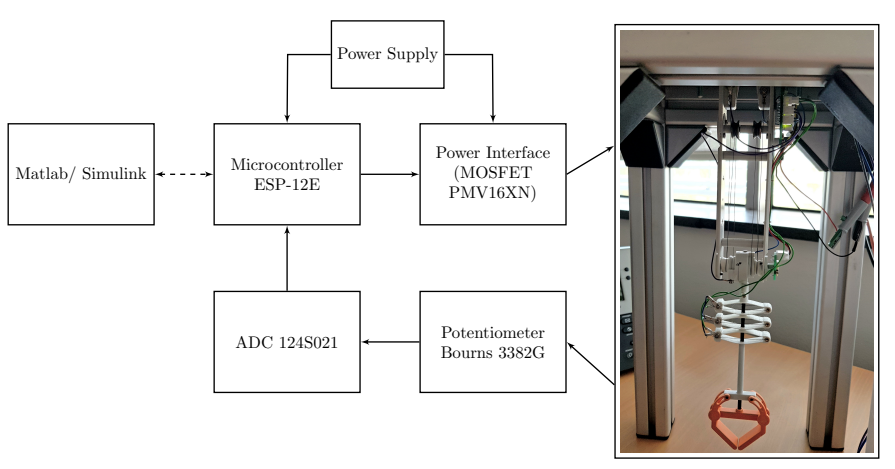

FIGURE 5: EXPERIMENTAL SETUP BLOCK DIAGRAM.

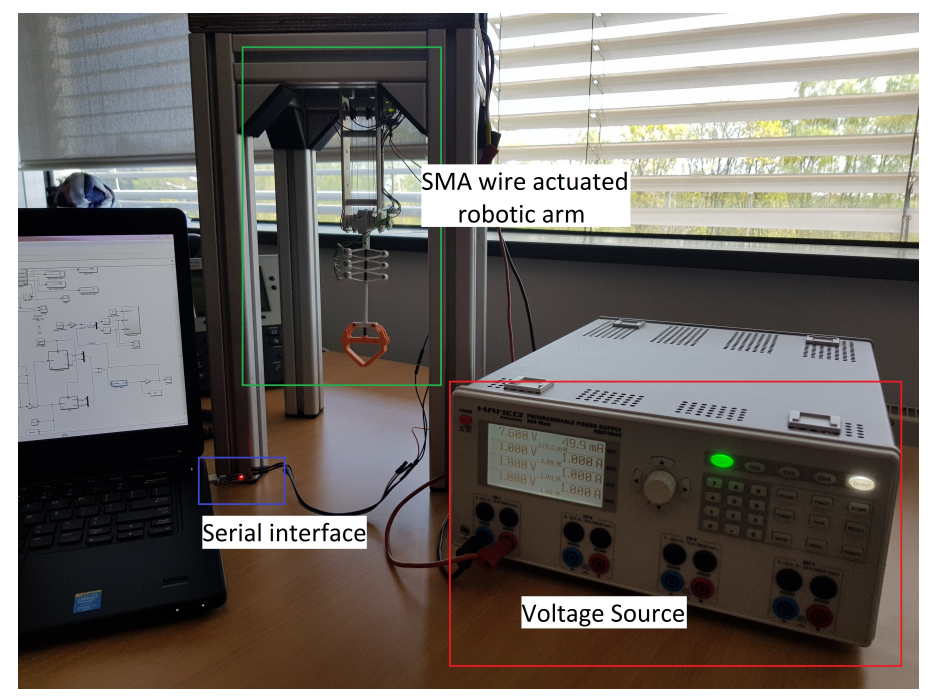

FIGURE 6: EXPERIMENTAL SETUP.

serial interface to a micro-controller ESP-12E, then processed into a PWM signal and applied to each wire through a MOSFET based power interface. The angular position is measured by a potentiometer (Bourne 3382G) in combination with a 12 bits ADC. The measurement is processed by the micro-controller and sent back to the Matlab/Simulink interface via serial communication. The SMA wires used for these experiments are from Dynalloy. Flexinol ${ }^{\circledR}$ wires of $0.31 \mathrm{~mm}$ diameter and $40 \mathrm{~cm}$ length are used for SMA-1 and SMA-2, while the gripper is actuated by a third Flexinol ${ }^{\circledR}$ wire of $0.15 \mathrm{~mm}$ diameter and $10 \mathrm{~cm}$ length. The two $7.5 \mathrm{~mm}$ diameter arm couplers are joined together by a torsion spring with a stiffness coefficient of $3.2 \mathrm{Nmm}$ per degree.

Both controllers were heuristically tuned to achieve the best possible regulation performance. The gains for the AC controller were set as follows: $\alpha_{1}=1, K_{1}=1.4$ and $\alpha_{2}=1, K_{2}=-1.4$. Similarly the gains for the PID control were set as: $K_{p 1}=2$,
$K_{i 1}=0.2, K_{d 1}=0.05$ and $K_{p 2}=-2, K_{i 2}=-0.05, K_{d 2}=-0.03$. The saturation voltage for both controllers is set from 0 to $4.5 \mathrm{~V}$. The controlled variable is the angular position of the robotic arm, thus the angular position of coupler- 1 . The position of this coupler is measured in relation with the negative $Z$-axis (see Fig. 1 ), where the zero position occurs when SMA-2 is at minimum strain, and the end-effector is over the negative $Z$ - axis (hanging down).

The controllers are tested for position regulation at three different desired positions. These desired positions are introduced as step signals at 0,20 and 40 seconds time with values of 16 , 25 and 12 degrees respectively (dashed line Fig. 7). The initial position of the robotic arm is the equilibrium point at ambient temperature and $0 \mathrm{~V}$ for both wires. It is important to notice that this initial position is different in each test. This difference, independently of the control approach, is due to the dependence of the wires on the pre-stress as well as previous states of the wires themselves. Thus the resting point at $0 \mathrm{~V}$ and ambient temperature changes slightly between each test. The experimental results for position regulation for both control approaches are shown in Fig. 7. The system was configured so that the control actions start 5 seconds after the data acquisition initial time $(\mathrm{t}=0)$. This initialization time allows the filter applied to the acquired data to stabilize before starting the control action.

Three different values were used to analyze and compare the performance of both controllers. These values are the average overshoot (OS), the average settling time during rising (ST+) and falling(ST-) references and finally the steady state error (SSE). Table 1 resumes these values for each control approach. The AC approach presents a slightly higher (2.06) OS in comparison with the PID (1.63 ). However, it is important to notice that the overshoot for each reference is only higher for the AC control during the first step. When comparing ST, the adaptive control has significantly faster ST with $3.4 \mathrm{~s}$ rising and $14.6 \mathrm{~s}$ falling references, while the PID control ST are 8.8 and 15.1 respectively. For the SSE the AC also presents better results with an SSE of 0.05 while the PID SSE is 0.083, these results are better shown in Fig. 8. In the overall, the adaptive control proved to be a better approach to deal with the nonlinear dynamics of the SMA wires.

Figure 9 shows the control voltage for both controllers AC (9a) and PID (9b). Here we can appreciate the voltage applied over SMA-1 and SMA-2 by each controller. However the voltage profiles are similar for AC and PID control, we can see a faster and higher response of the control voltage for AC controller, leading to a faster response and lower ST.

\section{CONCLUSIONS}

This paper presented the experimental validation for position regulation of an SMA actuated lightweight robotic arm using a direct adaptive control law. The robotic arm is actuated by a couple of antagonistic SMA wires, while a third SMA wire bia- 


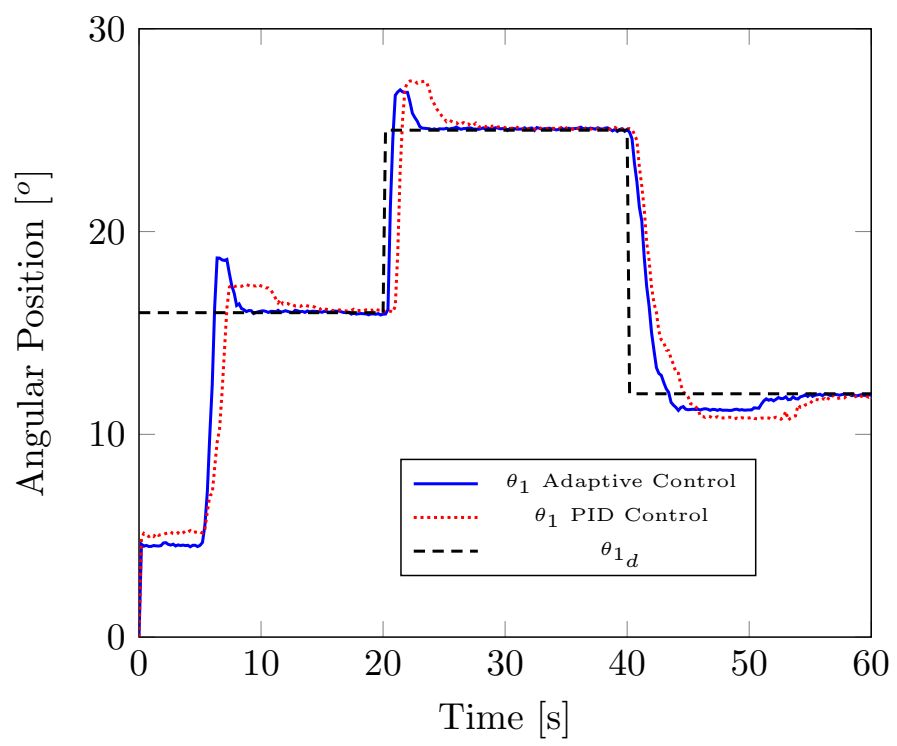

FIGURE 7: POSITION REGULATION WITH AC AND PID CONTROL.

TABLE 1: COMPARATIVE TABLE OF CONTROLLER PERFORMANCE.

\begin{tabular}{clllc}
\hline Control & ${\mathrm{OS}\left[{ }^{\circ}\right]}$ & $\mathrm{ST}+[\mathrm{s}]$ & $\mathrm{ST}-[\mathrm{s}]$ & $\mathrm{SSE}\left[{ }^{\circ}\right]$ \\
\hline PID & 1.63 & 8.8 & 15.1 & 0.083 \\
Adaptive & 2.06 & 3.4 & 14.6 & 0.05 \\
\hline
\end{tabular}

OS: Average Overshoot; ST: Average Settling Time (+/-: rising/falling step); SSE: Average Steady-State Error.

sed with a $3 \mathrm{D}$ printed custom spring actuates the gripper. The overall mechanism has an approximated weight of $50 \mathrm{~g}$ and a range of angular displacement up to 90 degrees. The design and mathematical model of the actuator were described. A direct adaptive control approach (AC) for angular position regulation was proposed and tested experimentally. These results were then compared with a classical PID control. After comparison of different aspects of the controller performance, such as overshoot, settling time and steady-state error, the adaptive control proved to deal better with the hysteretic nonlinear dynamic of the SMA wires. In future work, the response of the AC approach will be tested for disturbance rejection. Also, an observer-based controller will be implemented in order to achieve a faster and more accurate control performance.

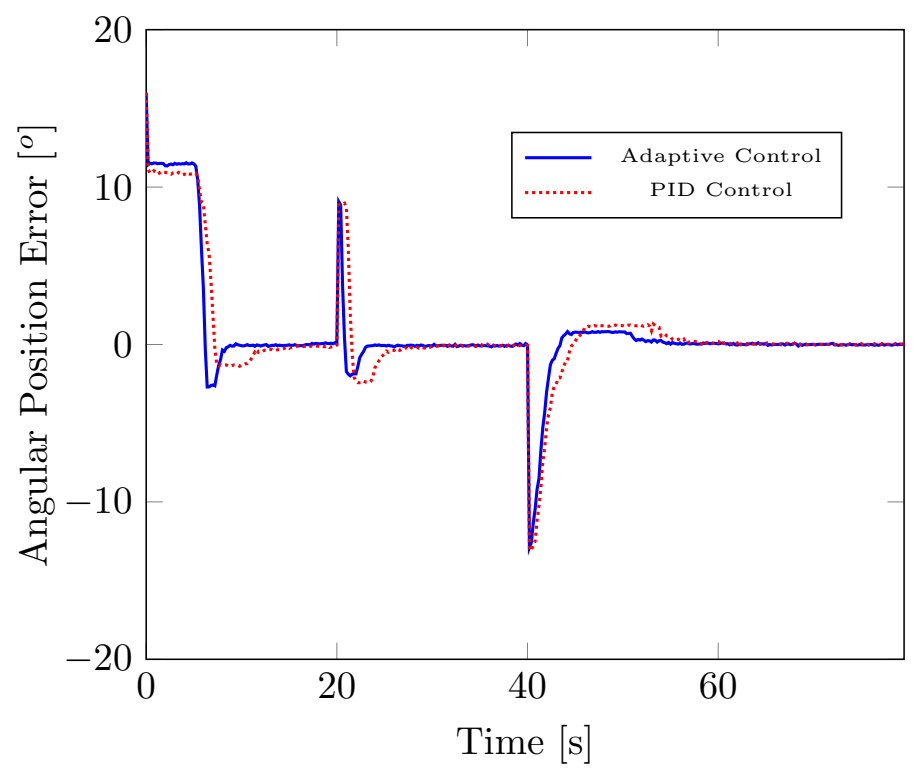

FIGURE 8: POSITION REGULATION ERROR WITH AC AND PID CONTROL.

\section{REFERENCES}

[1] Zheng, T., Yang, Y., Branson, D. T., Kang, R., Guglielmino, E., Cianchetti, M., Caldwell, D. G., and Yang, G., 2014. "Control design of shape memory alloy based multi-arm continuum robot inspired by octopus". In 2014 IEEE 9th Conference on Industrial Electronics and Applications (ICIEA), pp. 1108-1113.

[2] Rao, A., Srinivasa, A. R., and Reddy, J. N., 2015. Design of Shape Memory Alloy (SMA) Actuators. SpringerBriefs in Computational Mechanics. Springer International Publishing.

[3] Nematzadeh, F., and Sadrnezhaad, S., 2012. "Effects of material properties on mechanical performance of nitinol stent designed for femoral artery: Finite element analysis". Scientia Iranica, 19(6), pp. 1564 - 1571.

[4] Mataee, M. G., Andani, M. T., and Elahinia, M., 2015. "Adaptive anklefoot orthoses based on superelasticity of shape memory alloys". Journal of intelligent material systems and structures, 26(6), pp. 639-651.

[5] Moghaddam, N. S., Skoracki, R., Miller, M., Elahinia, M., and Dean, D., 2016. "Three dimensional printing of stiffness-tuned, nitinol skeletal fixation hardware with an example of mandibular segmental defect repair". Procedia CIRP, 49, Aug, pp. 45-50.

[6] Pandis, N., and Bourauel, C. P., 2010. "Nickel-titanium (niti) arch wires: the clinical significance of super elasticity". Seminars in Orthodontics, 16(4), pp. 249-257. Latest Controversies in Orthodontics: From in Vitro Data to in 


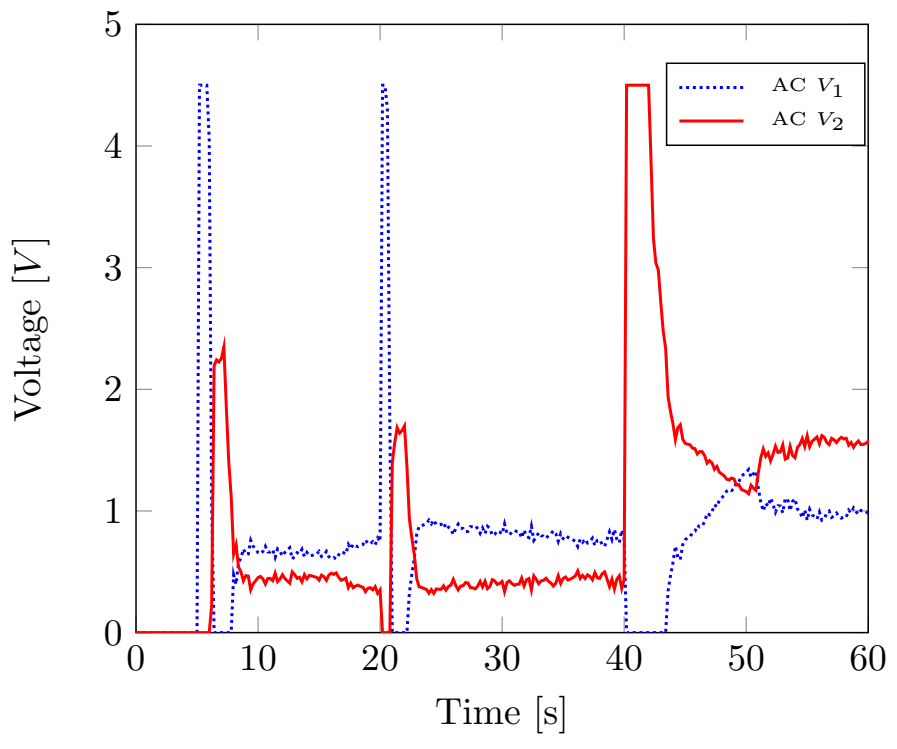

(a) ADAPTIVE CONTROL VOLTAGE

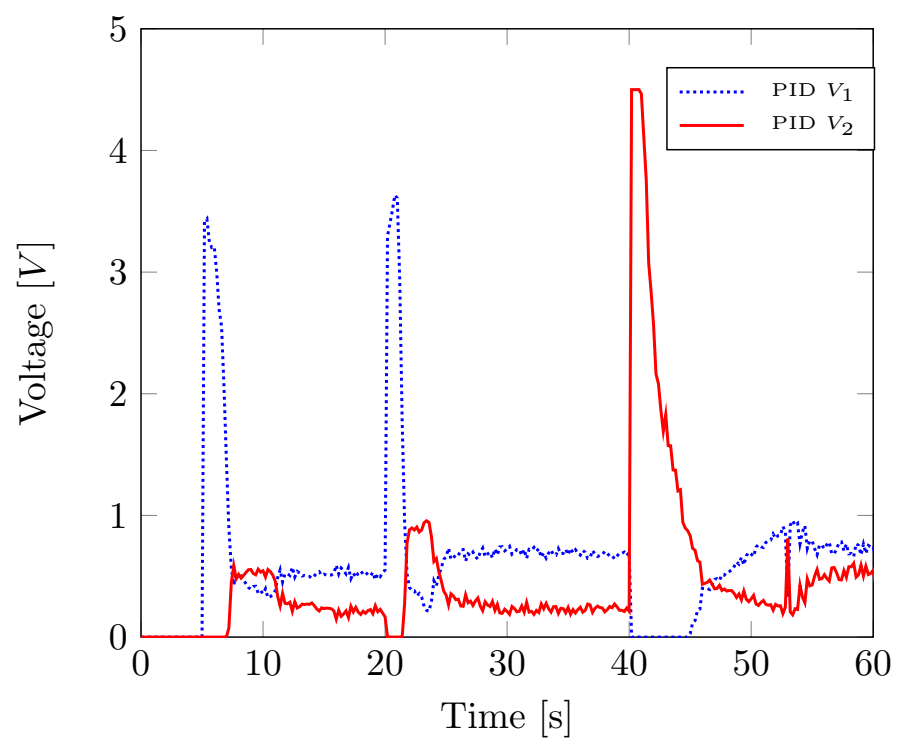

(b) PID CONTROL VOLTAGE

FIGURE 9: CONTROL VOLTAGE FOR AC AND PID CONTROLLERS.

Vivo Evidence.

[7] Rodrigue, H., Cho, S., Han, M.-W., Bhandari, B., Shim, J.E., and Ahn, S.-H., 2016. "Effect of twist morphing wing segment on aerodynamic performance of uav". Journal of Mechanical Science and Technology, 30(1), pp. 229-236.

[8] Barbarino, S., Ameduri, S., Lecce, L., and Concilio, A., 2009. "Wing shape control through an sma-based device". Journal of intelligent material systems and struc- tures, 20(3), pp. 283-296.

[9] Son, H.-M., Kim, M. Y., and Lee, Y.-J., 2009. “Tunablefocus liquid lens system controlled by antagonistic winding-type sma actuator". Optics Express, 17(16), jul, pp. 14339-14350.

[10] Williams, E. A., Shaw, G., and Elahinia, M., 2010. "Control of an automotive shape memory alloy mirror actuator". Mechatronics, 20(5), pp. 527-534.

[11] Quintanar-Guzmán, S., Reyes-Reyes, J., and ArellanoSánchez, M. d. c., 2014. "Modelado y control de un sistema electrotérmico-mecánico móvil basado en alambres musculares". In XVI Congreso Latinoamericano de Control Automático, CLCA 2014, Asociación de México de Control Automático, pp. 834-839.

[12] Hulea, M., and Caruntu, C. F., 2014. "Spiking neural network for controlling the artificial muscles of a humanoid robotic arm". In 2014 18th International Conference on System Theory, Control and Computing (ICSTCC), IEEE, pp. 163-168.

[13] Ko, J., Jun, M. B., Gilardi, G., Haslam, E., and Park, E. J., 2011. "Fuzzy pwm-pid control of cocontracting antagonistic shape memory alloy muscle pairs in an artificial finger". Mechatronics, 21(7), pp. 1190-1202.

[14] van der Elst, L. A., Quintanar-Guzmn, S., and HadjiMinaglou, J. R., 2017. "Design of an electromechanical prosthetic finger using shape memory alloy wires". In 2017 IEEE International Symposium on Robotics and Intelligent Sensors (IRIS), pp. 56-61.

[15] Kannan, S., Giraud-Audine, C., and Patoor, E., 2009. "Control of shape memory alloy (sma) actuator using seriesparallel model reference adaptive control (mrac)". In ASME 2009 Conference on Smart Materials, Adaptive Structures and Intelligent Systems, American Society of Mechanical Engineers, pp. 441-450.

[16] Kannan, S., Giraud-Audine, C., and Patoor, E., 2010. "Laguerre model based adaptive control of antagonistic shape memory alloy (sma) actuator". In Proc. SPIE 7643, Active and Passive Smart Structures and Integrated Systems 2010, Vol. 7643, pp. 764307-764307-12.

[17] Kannan, S., Giraud-Audine, C., and Patoor, E., 2013. “Application of laguerre based adaptive predictive control to shape memory alloy (sma) actuator". ISA Transactions, 52(4), pp. $469-479$.

[18] Kannan, S., 2011. "Modélisation et commande dactionneurs à alliage à mémoire de forme". PhD thesis, l'École Nationale Supérieure d'Arts et Métiers.

[19] Quintanar-Guzmán, S., Kannan, S., Olivares-Mendez, M. A., and Voos, H., 2016. "Operational space control of a lightweight robotic arm actuated by shape memory alloy (sma) wires". In ASME 2016 Conference on Smart Materials, Adaptive Structures and Intelligent Systems, Vol. Volume 2: Modeling, Simulation and Control; Bio-Inspired 
Smart Materials and Systems; Energy Harvesting, American Society of Mechanical Engineers.

[20] Guo, Z., Pan, Y., Wee, L. B., and Yu, H., 2015. "Design and control of a novel compliant differential shape memory alloy actuator". Sensors and Actuators A: Physical, 225, apr, pp. 71-80.

[21] Kannan, S., Quintanar-Guzmán, S., Bezzaoucha, S., Olivares-Mendez, M. A., and Voos, H., 2016. "Adaptive control of hysteretic robotic arm in operational space". In Proceedings of the 5th International Conference on Mechatronics and Control Engineering, ICMCE '16, ACM, pp. 92-96.

[22] Kannan, S., Bezzaoucha, S., Quintanar-Guzmán, S., Olivares-Mendez, M. A., and Voos, H., 2016. "Adaptive control of robotic arm with hysteretic joint". In Proceedings of the 4th International Conference on Control, Mechatronics and Automation, ICCMA '16, ACM, pp. 46-50.

[23] Pan, Y., Guo, Z., Li, X., and Yu, H., 2017. "Outputfeedback adaptive neural control of a compliant differential sma actuator". IEEE Transactions on Control Systems Technology, $\boldsymbol{P P}(99)$, pp. 1-9.

[24] Mai, H., Song, G., and Liao, X., 2013. "Adaptive online inverse control of a shape memory alloy wire actuator using a dynamic neural network". Smart Materials and Structures, 22(1), p. 015001.

[25] Tai, N. T., and Ahn, K. K., 2012. "Output feedback direct adaptive controller for a sma actuator with a kalman filter". IEEE Transactions on Control Systems Technology, 20(4), July, pp. 1081-1091.

[26] Quintanar-Guzmán, S., Kannan, S., Aguilera-González, A., Olivares-Mendez, M. A., and Voos, H., 0. "Operational space control of a lightweight robotic arm actuated by shape memory alloy wires: A comparative study". Journal of Intelligent Material Systems and Structures, O(0).

[27] Quintanar-Guzmán, S., Kannan, S., Olivares Mendez, M. A., and Voos, H., 2016. "Lightweight robotic arm actuated by Shape Memory Alloy (SMA) Wires". In International Conference on Electronics, Computers and Artificial Intelligence.

[28] Liang, C., and Rogers, C. A., 1990. "One-dimensional thermomechanical constitutive relations for shape memory materials". Journal of intelligent material systems and structures, 1(2), pp. 207-234.

[29] Elahinia, M. H., and Ashrafiuon, H., 2002. "Nonlinear control of a shape memory alloy actuated manipulator". Journal of Vibration and Acoustics, 124(4), pp. 566-575.

[30] Queiroz, M. S., Dawson, D. M., Nagarkatti, S. P., and Zhang, F., 2000. Lyapunov-Based Control of Mechanical Systems. Springer. 